\title{
Hertz elastic dynamics of two colliding elastic spheres
}

\author{
Bo-Hua Sun ${ }^{1,2}$ \\ 1) College of Civil Engineering \&3 Institute of Mechanics and Technology \\ Xi'an University of Architecture and Technology, Xi'an 710055, China \\ email: sunbohua@xauat.edu.cn \\ ${ }^{2)}$ Faculty of Mechanical Engineering 83 Mechanics, Ningbo University, Ningbo 315211, \\ China
}

\begin{abstract}
This paper revisits a classic problem in physics - Hertz elastic dynamics of two colliding elastic spheres. This study obtains impact period in terms of hypergeometric function and successfully combines Deresiewicz's three segmental solutions into one single solution. Our numerical investigation confirms that Deresiewicz's inversion is a good approximation. As an essential part of this study, a general Maple code is provided.
\end{abstract}

Keywords: Hertz impact dynamics, exact solution, numerical solution, inversion, Maple

\section{INTRODUCTION}

Body impact is a phenomenon of collision between bodies, in which the two colliding elastic spheres, as shown in Fig. 1, is of a popular one $\mathrm{e}^{1-7}$. When bodies collide, they come together with some relative velocity at an initial point of contact. If it were not for the contact force that develops between them, the normal componen$t$ of relative velocity would result in overlap or interference near the contact point and this interference would increase with time. This reaction force deforms the bodies into a compatible configuration in a common contact surface that envelopes the initial point of contact ${ }^{6}$.
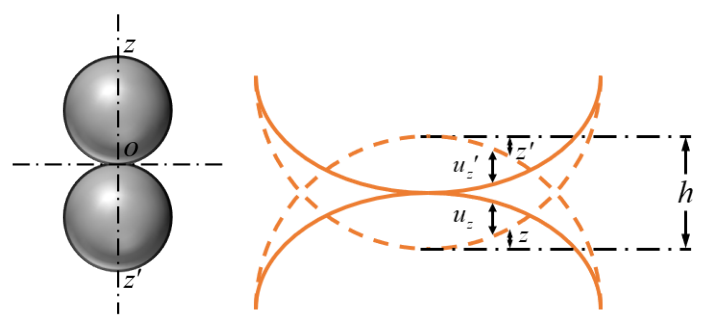

FIG. 1. Hertz law $P=k h^{3 / 2}$, stiffness $k=\frac{4}{5 E} \sqrt{\frac{R_{1} R_{2}}{R_{1}+R_{2}}}$, relative distance $h(t)$, radius of ball $R_{1}$ and $R_{2}$, mass of each ball $m_{1}, m_{2}$, reduce mass $M=\frac{m_{1} m_{2}}{m_{1}+m_{2}}$, effective modulus $E=\frac{3}{4}\left(\frac{1-\nu_{1}^{2}}{E_{1}}+\frac{1-\nu_{2}^{2}}{E_{2}}\right)$, the Young's modulus $E_{1}, E_{2}$, and Poisson's ratio $\nu_{1}, \nu_{2}$.

When bodies collide, they come together with some relative velocity at an initial point of contact. Hertz's theory of impact of elastic bodies with relative velocity $v$ leads to the energy integral ${ }^{2,4}$

$$
\mu \dot{h}^{2}+k h^{5 / 2}=M v^{2} .
$$

Love $^{2}$ obtained the duration of impact $T=$ $\frac{4 \sqrt{\pi} \Gamma(2 / 5)}{5 \Gamma(9 / 10)}\left(\frac{M^{2}}{k^{2} v}\right)^{1 / 5} \simeq 2.94\left(\frac{M^{2}}{k^{2} v}\right)^{1 / 5}=2.95 \frac{h_{0}}{v}$, where the maximum distance $h_{0}=\left(\frac{M v^{2}}{k}\right)^{2 / 5}$. Deresiewicz ${ }^{3}$ obtained the exact impact trajectory solution expressed in the incomplete beta function, namely $t(\xi)=2 h_{0} /(5 v) B(\xi ; 2 / 5,1 / 2)$.

However, the evaluation of $B(\xi ; 2 / 5,1 / 2)$ was made in three distinct intervals of the variables, namely (a) For $0<\xi \leq 0.4: t(\xi)=B(\xi ; 2 / 5,1 / 2)$, (b) For $0.4 \leq \xi \leq$ 0.75 were obtained numerical integration using Simpson's rule with internal 0.05 (c) For $0.75 \leq \xi<1$ : $t(\xi)=$ $B(1 ; 2 / 5,1 / 2)-B(1-\xi ; 1 / 2,2 / 5)$. The Deresiewicz three segmental solutions $t_{D}$ are listed below:

$$
t_{D}=\left\{\begin{array}{cc}
B\left(\xi ; \frac{2}{5}, \frac{1}{2}\right), & 0<\xi \leq 0.4 \\
\text { numerical integration, } & 0.4 \leq \xi \leq 0.75 \\
B\left(1 ; \frac{2}{5}, \frac{1}{2}\right)-B\left(1-\xi ; \frac{1}{2}, \frac{2}{5}\right), & 0.75 \leq \xi<1
\end{array}\right.
$$

Based on his own numerical results for the half duration of impact $t \in[0, T / 2]$, Deresiewicz ${ }^{3}$ proposed an approximate fitted inversion solution of $t(h)$

$$
h_{D} \approx h_{0} \sin \left(\frac{\pi t}{T}\right) .
$$

However, Deresiewicz ${ }^{3}$ had not verified the correctness of $h_{D}$ for another half duration of impact, namely $t \in$ $[T / 2, T]$.

In this short article, firstly we verify Deresiewicz's exact solution and find out its validation range. Then combine the three segmental solutions into one single solution. Once we have the single solution, we will propose an better approximate inversion solution.

\section{EXACT SOLUTION}

Introducing a transformation, $\xi=k h^{5 / 2} /\left(M v^{2}\right)$, with the help of symbolic software, Maple, we are able to find 
the solution of Eq.1 as follows

$$
\begin{aligned}
t & =\frac{1}{v} \int_{0}^{h} \frac{d h}{\sqrt{1-k h^{5 / 2} /\left(M v^{2}\right)}}=\frac{2}{5} \frac{h_{0}}{v} \int_{0}^{\xi} \frac{\xi^{-3 / 5} d \xi}{\sqrt{1-\xi}} \\
& =\frac{h_{0}}{v} \xi^{2 / 5} \text { hypergeom }\left(\left[\frac{2}{5}, \frac{1}{2}\right],\left[\frac{7}{5}\right], \xi\right)
\end{aligned}
$$

or in terms of $h$, we have

$$
t=\frac{h}{v} \operatorname{hypergeom}\left(\left[\frac{2}{5}, \frac{1}{2}\right],\left[\frac{7}{5}\right], \frac{k}{M v^{2}} h^{5 / 2}\right),
$$

where hypergeom is the hyper-geometrical function. Hence, the duration of impact is given by $T=2 t(1)=$ $\frac{4}{5} B\left(\frac{2}{5}, \frac{1}{2}\right) \frac{h_{0}}{v}=2.943275184 \frac{h_{0}}{v}$, which was obtained by Love $^{2}$. The solution in Eq.4 is shown in Fig. 2 below.

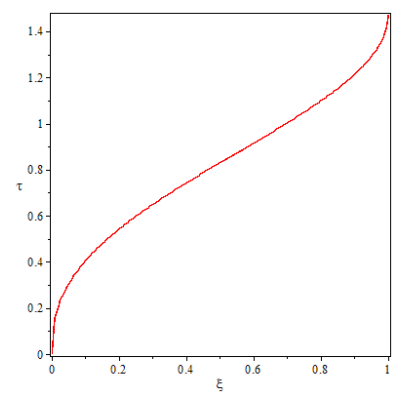

FIG. 2. $\tau=t /\left(h_{o} / v\right)=\tau(\xi)$

Similar to Deresiewicz ${ }^{3}$, we define a time ratio as follows

$$
\frac{t}{(T / 2)}=\frac{\frac{h_{0}}{v} \xi^{2 / 5} \text { hypergeom }\left(\left[\frac{2}{5}, \frac{1}{2}\right],\left[\frac{7}{5}\right], \xi\right)}{(T / 2)}
$$

The comparison of the time ratio is illustrated in both Table II and Fig. 3 below.

TABLE I. Numerical results of $\frac{t}{(T / 2)}$

\begin{tabular}{ccc}
\hline \hline$\xi$ & $\frac{t}{(T / 2)}\left(\right.$ Deresiewicz $\left.^{3}\right)$ & $\frac{t}{(T / 2)}$ from Eq.5 \\
\hline 0.001 & 0.0429 & 0.04288063339 \\
0.01 & 0.108 & 0.1078504177 \\
0.1 & 0.275 & 0.2745641588 \\
0.2 & 0.368 & 0.3681657644 \\
0.3 & 0.441 & 0.4406836100 \\
0.4 & 0.504 & 0.5041630552 \\
0.5 & 0.563 & 0.5634585666 \\
0.6 & 0.622 & 0.6215676756 \\
0.7 & 0.681 & 0.6812060523 \\
0.75 & 0.713 & 0.7126658744 \\
0.8 & 0.746 & 0.7460966450 \\
0.9 & 0.824 & 0.8244806367 \\
1.0 & 1.0 & 1.0 \\
\hline \hline
\end{tabular}

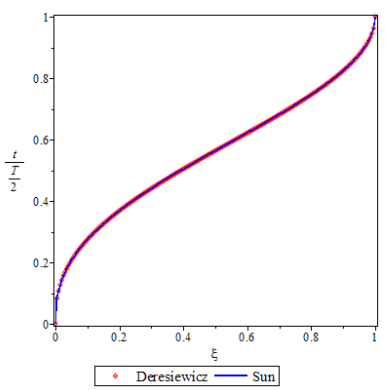

FIG. 3. Red point is Deresiewicz's solution ${ }^{3}$, the blue line is from Eq.5. They are perfectly agree each other.

From both Table II and Fig.3, it is delighted to see that the solution in Eq.4 and Eq.5 produce the same results as the three segmental solutions of Deresiewicz ${ }^{3}$, which reveals that the three segmental solutions in Eq.2 can be combined into a single solution as in Eq.4 and or Eq.5. In other words, the solution in Eq.4 is valid for the whole domain of $\xi \in[0,1]$.

However, since the solution in Eq.4 is monotonic, the solution only works in the domain of $t \in[T / 2, T]$, and can not predict the value of $t=t(\xi)$ in another half domain $t \in[T / 2, T]$.

\section{NUMERICAL SOLUTION AND MAPLE CODE}

To get a solution for the full duration of impact, ie., $t \in[T / 2, T]$, numerical method must be adopted. For reader's ease teaching and research, a general Maple code is provided below.

TABLE II. A general Maple code of the Hertz impact

\begin{tabular}{c|c}
\hline \hline Item & Maple code \\
\hline Equation & eq $:=\operatorname{diff}(\mathrm{h}(\mathrm{t}), \mathrm{t}, \mathrm{t})+(5 / 4)^{*} \mathrm{k}^{*} h(t)^{3 / 2} / \mathrm{M}=0$ \\
Initial condition & ins $:=\mathrm{h}(0)=0,(\mathrm{D}(\mathrm{h}))(0)=\mathrm{v} ;$ \\
Solution & sol $:=\operatorname{dsolve}(\mathrm{eq}, \mathrm{ins}$, numeric); \\
Plot & with(plots); odeplot $(\mathrm{sol},[\mathrm{t}, \mathrm{h}(\mathrm{t})], \mathrm{t}=0 . . \mathrm{T})$ \\
\hline \hline
\end{tabular}

A numerical example is shown in Fig.4 below.

\section{SOLUTION INVERSION}

The solution in Eq.4 can be expanded in Taylor's series as follows

$$
t=\frac{h_{0}}{v} \sum_{0}^{n} \frac{(2 n) !}{\left(n+\frac{2}{5}\right) 4^{n}(n !)^{2}} \xi^{n+\frac{2}{5}}
$$




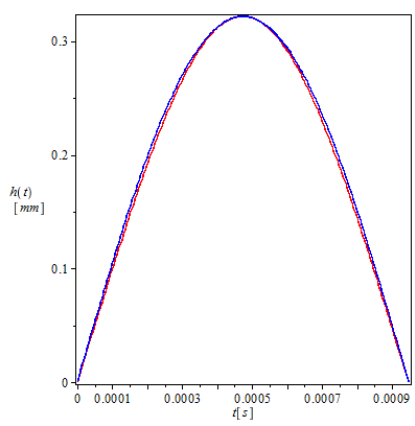

FIG. 4. Data taken from page 128, Stronge $^{6}: \mathrm{m}[1]:=32.4[\mathrm{~kg}]$; $\mathrm{m}[2]:=32.4[\mathrm{~kg}] ; \mathrm{R}[1]:=0.1 \mathrm{e}-1[\mathrm{~m}] ; \mathrm{R}[2]:=0.1 \mathrm{e}-1[\mathrm{~m}] ; \mathrm{E}[1]:=$ $0.210 \mathrm{e} 12[\mathrm{~N} / \mathrm{mm}] ; \mathrm{E}[2]:=0.210 \mathrm{e} 12[\mathrm{~N} / \mathrm{mm}] ; \mathrm{nu}[1]:=.3 ; \mathrm{nu}[2]:=.3 ;$ $\mathrm{M}:=\mathrm{m}[1]^{*} \mathrm{~m}[2] /(\mathrm{m}[1]+\mathrm{m}[2]) ; \mathrm{E}:=3 / 4^{*}\left(\left(1-\mathrm{nu}[1]^{*} \mathrm{nu}[1]\right) / \mathrm{E}[1]+(1-\right.$ $\mathrm{nu}[2] * \mathrm{nu}[2]) / \mathrm{E}[2]) ; \mathrm{k}:=4^{*} \operatorname{sqrt}(\mathrm{R}[1] * \mathrm{R}[2] /(\mathrm{R}[1]+\mathrm{R}[2])) /(5 * \mathrm{E}) ; \mathrm{v}:=$ $1[\mathrm{~m} / \mathrm{s}] ; \quad \mathrm{T}:=2.943275184 *(M * M /(k * k * v))(1 / 5)$. Red-line is our numerical result, Blue-line is Deresiewicz's approximation $h_{D} \approx h_{0} \sin \left(\frac{\pi t}{T}\right)$. It is surprised to see that the results are agree with each other very well.

Generally speaking, the inversion $\xi=\xi(t)$ is hard to be found. Therefore, Deresiewicz's approximation $h_{D}$ in Eq.3 is a remarkable inversion of the series in Eq.7. Although Deresiewicz mentioned that $h_{D}$ in Eq.3 was obtained from his data fitting, to get such elegant and accuracy expression, I guess that Deresiewicz might got some ideas from the Taylor'series of $\arcsin x=$ $\sum_{0}^{n} \frac{(2 n) !}{(2 n+1) 4^{n}(n !)^{2}} x^{2 n+1}$.

\section{CONCLUSIONS}

In conclusion, this short article successfully combined Deresiewicz's three segmental solutions into a single function, and shown that the Deresiewicz's inversion is a high accuracy approximation. A numerical example is carried out and a general Maple code is provided.

Availability of data: There is no additional data in this study.

${ }^{1}$ H. Hertz, Ueber die Beruhrung fester elastischer Körper, GrelleBorchardt, XCII, 1882.

${ }^{2}$ Love, A.E.H., Mathematical Theory of Elasticity. 4th. Ed., art.139, Cambridge: Cambridge University Press, 1927.

${ }^{3}$ Deresiewicz, H., A note on Hertz's theory of impact, Acta Mechanica, 6:110-112, 1968.

${ }^{4}$ Landau, L.D. and Lifshitz, E.M., Theory of Elasticity. 3rd ed. Butterworth-Heinemann, 1986.

${ }^{5}$ Johnson K.L. Contact Mechanics. Cambridge: Cambridge University Press, 1987.

${ }^{6}$ Stronge, W.J. Impact Mechanics. Cambridge: Cambridge University Press, 2000.

${ }^{7} \mathrm{Yu}$, T.X. A puzzle: how big is the colliding force? Mechanics in Engineering, 40(3):344-348, 2018. 